\title{
Numerical solution for the fluid flow between active elastic walls
}

\author{
F. Z. Ahmed ${ }^{1} \quad$ D. V. Strunin ${ }^{2} \quad$ M. G. Mohammed ${ }^{3}$ \\ R. P. Bhanot ${ }^{4}$
}

(Received 9 January 2016; revised 16 September 2016)

\begin{abstract}
We analyse a model of the fluid flow between elastic walls simulating arteries actively interacting with the blood. Lubrication theory for the flow is coupled with the pressure and shear stress from the walls. The resulting nonlinear partial differential equation describes the displacement of the walls as a function of the distance along the flow and time. The equation is solved numerically using the one-dimensional integrated radial basis function network method. A solution in the form of a self-sustained train of pulses is obtained. Numerical experiments demonstrate the process of formation of the train from randomly chosen initial conditions. Dependence of the pulses on the boundary conditions is explored.
\end{abstract}

DOI:10.21914/anziamj.v57i0.10453, (c) Austral. Mathematical Soc. 2016. Published October 10, 2016, as part of the Proceedings of the 12th Biennial Engineering Mathematics and Applications Conference. ISSN 1445-8810. (Print two pages per sheet of paper.) Copies of this article must not be made otherwise available on the internet; instead link directly to the DOI for this article. Record comments on this article via http://journal. austms.org. au/ojs/index.php/ANZIAMJ/comment/add/10453/0 


\section{Contents}

1 Introduction

C222

2 Pulses as auto-waves

C223

3 Numerical approach

C227

4 Numerical experiments

C228

5 Conclusion

C231

References

C233

\section{Introduction}

Mathematical models of arterial blood flows typically treat arteries as passive material [1, 2]. A popular approximation of the flow-artery interaction is the proportionality between the increment in the artery's cross-sectional area and the flow pressure, $p-p_{0} \sim \sqrt{A}-\sqrt{A_{0}}$, where $p_{0}$ and $A_{0}$ are the reference pressure and cross-sectional area, respectively [3]. Studies of pulse propagation then focus on passive response to the pre-formed time-periodic boundary condition at the artery's inlet [4].

Very few models assume that the arteries actively exert pressure [5]. These models are non-autonomous, that is, the active component of the pressure is introduced in the form of a prescribed function of time and coordinate. This renders the pulses a result of an unexplained external factor.

Previously we formulated an autonomous pulse model describing the hypothetical flow between active elastic walls [6]. The geometry of the flow is the channel between infinitely stretching elastic walls rather than tubes. The flow dynamics are governed by a partial differential equation partly constructed on 
phenomenological principles. The model cannot be directly applied to actual arteries. However, it is based on the physical idea of the balance between forces which play the key role in the pulse propagation: the push from the walls and viscous friction.

Thus, from a dynamical point of view our model is active-dissipative and the pulses are auto-waves, that is, self-sustained dissipative structures. Unlike conservative waves, for example waves on fluid surface in gravity, the autowaves have unique speed and unique amplitude.

\section{Pulses as auto-waves}

Previously we formulated an auto-wave model for spinning combustion fronts [7]

$$
\partial_{t} F=\partial_{x}^{6} F-\partial_{x}\left[\left(\partial_{x} F\right)^{3}\right]+\left(\partial_{x} F\right)^{4},
$$

where $F(x, t)$ stands for the distance passed by the front (a line between unburned and burned mixture) along a cylinder as a function of the transversal coordinate $x$ and time $t$. The function $F(x, t)$ is subject to periodic boundary conditions. This system is active-dissipative: its active nature is due to the heat released from chemical reactions, and its dissipative nature is due to heat conductivity.

On differentiating equation (1) by $x$ and defining $\partial_{x} F=W$ we obtain

$$
\partial_{t} W=\partial_{x}^{6} W-\partial_{x}^{2}\left(W^{3}\right)+\partial_{x}\left(W^{4}\right) .
$$

The spatially uniform solution $F=$ const of (1) and corresponding uniform solution $W \equiv 0$ of (2) are stable under small perturbations as the linearised equation $\partial_{t} F=\partial_{x}^{6} F$ is purely dissipative.

For compactness, from now on we use primes/Roman numerals to denote derivatives with respect to $x$. 
To explain the mechanism of (1), denote the typical amplitude of the perturbation of the uniform solution by $\Delta \mathrm{F}>0$ and typical spatial scale of the perturbation by $\Delta x>0$. If the perturbation is sufficiently large, then it starts to grow due to the pumping effect of the (third order) nonlinear excitation, $-\left(\mathrm{F}^{\prime 3}\right)^{\prime}=-3 \mathrm{~F}^{\prime 2} \mathrm{~F}^{\prime \prime}$. This term is interpreted as nonlinear anti-diffusion. Let us evaluate the terms by the order of magnitude. We have in absolute value

$$
\left(\mathrm{F}^{\prime 3}\right)^{\prime} \sim(\Delta \mathrm{F})^{3} /(\Delta \mathrm{x})^{4} .
$$

As the perturbation grows, the higher order nonlinearity comes into play,

$$
\left(\mathrm{F}^{\prime}\right)^{4} \sim(\Delta \mathrm{F})^{4} /(\Delta \mathrm{x})^{4} .
$$

This nonlinearity makes side slopes of the $F$ shape steeper (the $W$ profile locally surges in amplitude) with small $\Delta x$. On those steep sections the dissipation prevails because it has higher order in $\Delta x$,

$$
\mathrm{F}^{\mathrm{VI}} \sim \Delta \mathrm{F} /(\Delta \mathrm{x})^{6} .
$$

As a result, the steep sections are prevented from developing into singularities. Instead, smooth self-sustained dissipative structures are formed, as shown in Figure 1. Each individual pulse is essentially a stable auto-soliton with the amplitude and speed controlled by the governing equation.

It is well established that in fluid flows between elastic walls, the dissipation is represented by the sixth order spatial derivative $[8,9]$. This brings about an idea to extend the model formulated by Huang and Suo [8] by excitation terms representing active motion of the elastic walls. On this foundation we construct the following model, which is structurally similar to (2) [6],

$$
\begin{aligned}
\partial_{\mathrm{t}} w= & \frac{\mathrm{D}}{3 \eta}\left(\mathrm{H}^{3} w^{v}\right)^{\prime}-\frac{E h}{6 \eta\left(1-v^{2}\right)}\left[\mathrm{H}^{3}\left(w^{\prime 3}\right)^{\prime \prime}\right]^{\prime} \\
& -\frac{1}{3 \eta} \alpha\left[\mathrm{H}^{3}\left(w^{4}\right)^{\prime}\right]^{\prime}+\beta\left(w^{5}\right)^{\prime} .
\end{aligned}
$$


Figure 1: A train of kink-shaped (F) and pulse-shaped (W) auto-waves. The Fwave moves upwards and to the left; the $\mathrm{W}$-wave moves horizontally to the left.
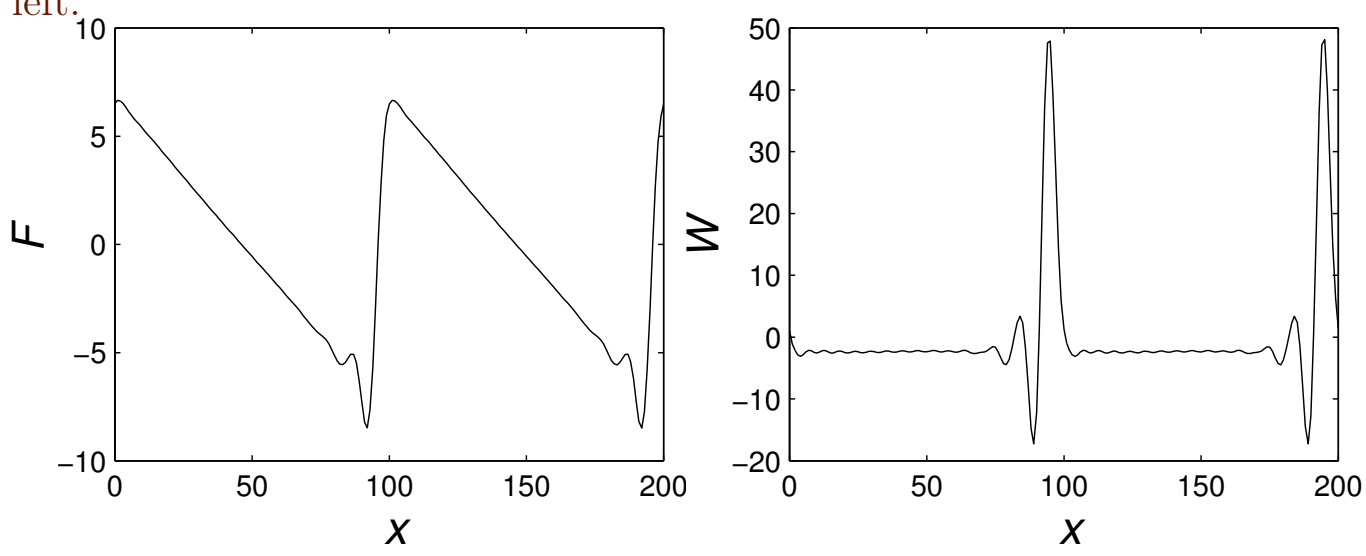

In PDE (3) $\mathrm{H}(x, t)$ is the half-width of the channel, $x$ the coordinate along the flow, $w(x, t)$ the transversal displacement of the wall counted from the neutral position, $z=\mathrm{H}_{0}(z$ is directed across the flow $)$, so that $\mathrm{H}=\mathrm{H}_{0}+w$, $\mathrm{D}$ is the flexural rigidity of the wall, $\mathrm{E}$ is Young's modulus, $\mathrm{h}$ is the thickness of the wall, $v$ is Poisson's ratio, $\eta$ is the viscosity, and $\alpha$ and $\beta$ are empirical parameters.

Equation (3), written in terms of $\boldsymbol{f}$ defined by $w=f^{\prime}$, becomes

$$
\partial_{t} f=\frac{D}{3 \eta} H^{3} f^{V I}-\frac{E h}{6 \eta\left(1-v^{2}\right)} H^{3}\left[\left(f^{\prime \prime}\right)^{3}\right]^{\prime \prime}-\frac{1}{3 \eta} \alpha H^{3}\left[\left(f^{\prime}\right)^{4}\right]^{\prime}+\beta\left(f^{\prime}\right)^{5}+C,
$$

where $\mathrm{C}$ is the constant of integration. The constant is eliminated by the change $f \rightarrow f+C t$, so we assume $C=0$. The first two terms on the right-hand side of (4) follow from the classical theory and are therefore dissipative.

Comparing (4) with (1), notice the higher order of nonlinearity of the excitation (fourth instead of third); this is necessary to overpower the third order classical term in (4). Accordingly, the last term in (4) has an even higher fifth 
order of nonlinearity, required to counterbalance the excitation. Evaluate the terms by the order of magnitude in absolute value. The excitation is

$$
\frac{1}{3 \eta} \alpha H^{3}\left[\left(f^{\prime}\right)^{4}\right]^{\prime} \sim\left(f^{\prime}\right)^{3} f^{\prime \prime} \sim(\Delta f)^{4} /(\Delta x)^{5} .
$$

As the amplitude of the perturbation increases, the fifth order nonlinearity comes into play,

$$
\beta\left(f^{\prime}\right)^{5} \sim(\Delta f)^{5} /(\Delta x)^{5} .
$$

This nonlinearity steepens the profile of $f$ at certain sections where the dissipation eventually prevails due to the higher order in $\Delta x$,

$$
\frac{\mathrm{D}}{3 \eta} \mathrm{H}^{3} \mathrm{f}^{\mathrm{VI}} \sim \Delta \mathrm{f} /(\Delta \mathrm{x})^{6} .
$$

This mechanism is similar to that of (1), so the pulse waves should exist in the hydro-elastic model (3).

Unlike the combustion model (2), the hydro-elastic model should generate only pulses travelling to the left. This is caused by the asymmetry: the excitation term produces energy only on the sections with positive slopes, $f^{\prime}>0$, where $-\left(f^{\prime}\right)^{3} f^{\prime \prime}$ is indeed the (nonlinear) anti-diffusion. However, on the negative slopes, $f^{\prime}<0$, the model acts as normal (yet nonlinear) diffusion. We are satisfied with this property as it implies that our quasi-artery differentiates between the direction to and from the 'heart'. The direction to the heart is to the right in Figure 1, accordingly the pulses go in the opposite direction, that is, to the left.

Our last remark in this section concerns the coefficients $\alpha$ and $\beta$. These parameters are empirical; however, having only two empirical parameters is a rather positive feature because, when modelling a biosystem, the more details one takes into account the more coefficients need to be involved. Then the difficult issue of how to determine their values arises. With this in mind, a model with only two empirical parameters is quite attractive. 


\section{$3 \quad$ Numerical approach}

To solve equation (4) we developed numerical codes based on the onedimensional Integrated Radial Basis Function Networks (1D-IRBFN) method. This method was tested in many engineering problems including viscous flows [10] and structural analysis [11]. It was demonstrated that the 1DIRBFN method has advantages over other numerical methods, for example finite difference and finite element methods, in terms of accuracy, faster approach and efficiency [12].

In the 1D-IRBFN method the highest order derivative in a differential equation is approximated by radial basis functions (RBFs) and, further, the lower order derivatives and function itself are then obtained by integration. The purpose of using integration to construct the approximants is to avoid the reduction in convergence rate caused by differentiation and also to improve the numerical stability of a discrete solution. The integration process naturally gives rise to arbitrary constants that serve as additional expansion coefficients. Therefore the constants facilitate the employment of some extra equations in the process of converting the RBF weights into the function values, which helps in the implementation of multiple boundary conditions. The RBFs yield better accuracy, are easy to implement and have the capability to provide a very accurate solution using relatively low numbers of grid points. The so-called multiquatric functions were found to be the most efficient basis function to use in the method [12]. The IRBFN ability to capture sharp gradients enables us to effectively investigate equation (4). For time integration we used the second order Crank-Nicolson method. 


\section{Numerical experiments}

In this section we report the numerical results on equation (4) presented in nondimensional form as

$$
\partial_{t} f=A f^{V I}-B\left[\left(f^{\prime \prime}\right)^{3}\right]^{\prime \prime}-C\left[\left(f^{\prime}\right)^{4}\right]^{\prime}+F\left(f^{\prime}\right)^{5} .
$$

The main question that we want to answer is whether the equation has the desired pulse-like solutions. Determining the values of the (dimensional) coefficients present in (3)-(4) may not be easy, this especially concerns the empirical coefficients $\alpha$ and $\beta$; we plan this as a separate study. In this article we arbitrarily select the values of the coefficients in the nondimensional form (5) just to demonstrate that the desired solutions exist.

Figure 2 shows the settling of a fixed-shaped travelling front evolving from a step-like initial condition. The boundary conditions set zero values of the first three derivatives of $f$ on the left edge and on the right edge of the domain. Observe how the step grows from the initial amplitude of around 2 to 5 ; this eventual size is dictated by the equation, not the initial condition. Notice the little cavity ahead of the main front - this cavity is an anticipated feature caused by the high order dissipation. The left-most line in this experiment corresponds to the moment when the main front is just about to hit the left boundary. The shape of the front has already settled by this time. If the left edge was located farther to the left in this experiment, giving more space for the front to propagate, then the front would continue to move with constant velocity. The $w$-plot shows the settling of the pulse and its steady propagation to the left.

Figure 3 presents the dynamics originating from the initial condition that is a mirror image of the initial condition in the experiment shown in Figure 2. The initial step has the same vertical size but has opposite orientation: it subsides from the left to the right. This is the way we configured it so as to 'help' the front move to the right, rather than to the left as in the previous experiment of Figure 2. To our satisfaction, the right-directed front motion 
Figure 2: $A=1, B=0.167, C=1.625, F=0.05$. The left-most line corresponds to $t=39$. The initial condition is $f(x, 0)=1.2 \tanh (x+5)$. The number of grid points is 100 , time step is 0.001 .
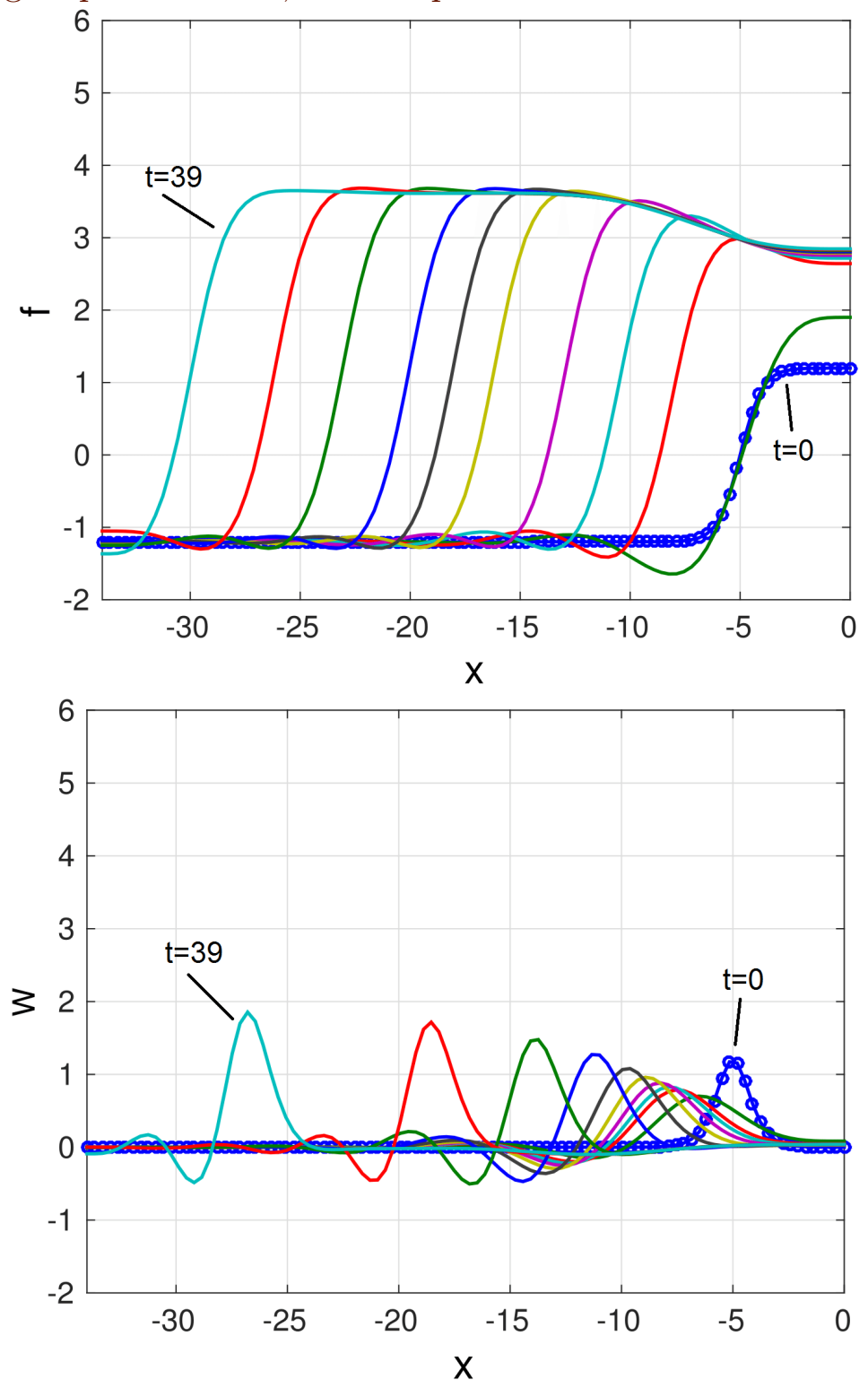
Figure 3: $\mathrm{A}=1, \mathrm{~B}=0.167, \mathrm{C}=1.625, \mathrm{~F}=0.05$. The lowest line corresponds to $t=39$. The initial condition is $f(x, 0)=1.2 \tanh (-x)$. The number of grid points is 100 , time step is 0.001 .

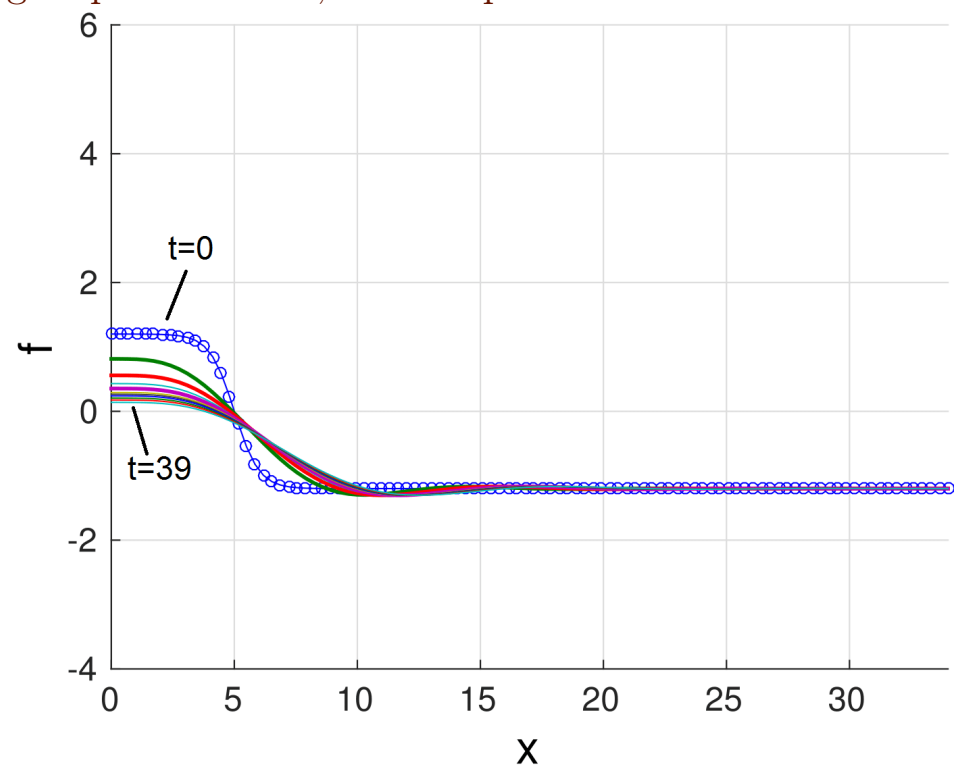

never eventuates, in agreement with the qualitative analysis of the governing equations (4)-(5). As predicted, the dynamics decay due to the lack of energy supply.

The second group of numerical experiments use periodic boundary conditions. The solution is assumed periodic in space with a prescribed value of the period. As equation (4) is of sixth order, the first five derivatives of the function $\mathbf{f}$ are also assumed periodic. The experiment displayed in Figure 4 starts from the initial condition in the form of two peaks. Over time the peaks evolve into the two-step settled configuration. This represents two pulses following one another, as is seen in Figure 5. 
Figure 4: $A=1, B=0.167, C=977.4, F=9720$. The initial condition is $f(x, 0)=0.2 \sin (x / 2)$. The shown profiles are for $t=0,9,13$.

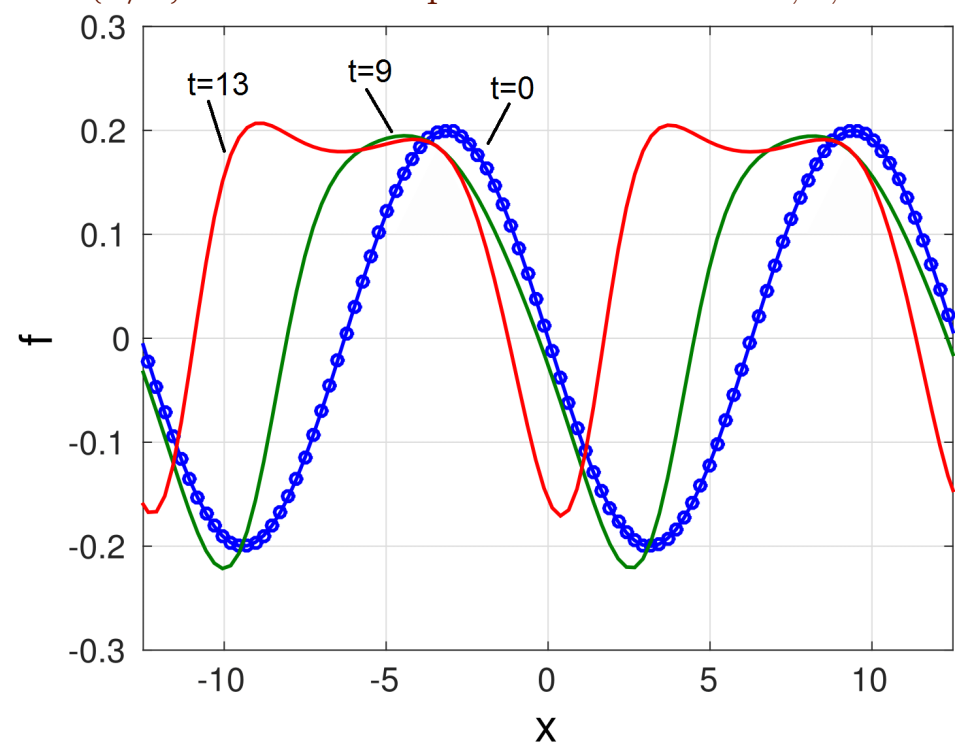

\section{Conclusion}

We presented an autonomous model, without claim of a direct link to biology at this stage, of a hypothetical flow between active elastic walls. Lubrication theory is used for the flow, and the walls are supposed to actively exert pressure and shear stress. The analogy with the combustion front equations indicates that the model should have auto-wave solutions in the form of pulses. We developed the numerical code solving the model using the one-dimensional integrated radial basis function network (1D-IRBFN) method. In line with expectations the computations gave the trains of pulses travelling in selfsustained fashion in the preferred direction. The two-pulse settled regime is obtained when the initial condition has the proper form to seed two pulses. 
Figure 5: The settled regime for the experiment shown in Figure 4. The snapshot times are $t=35,35.05,35.1,35.15,35.9,35.95,36,36.05$.
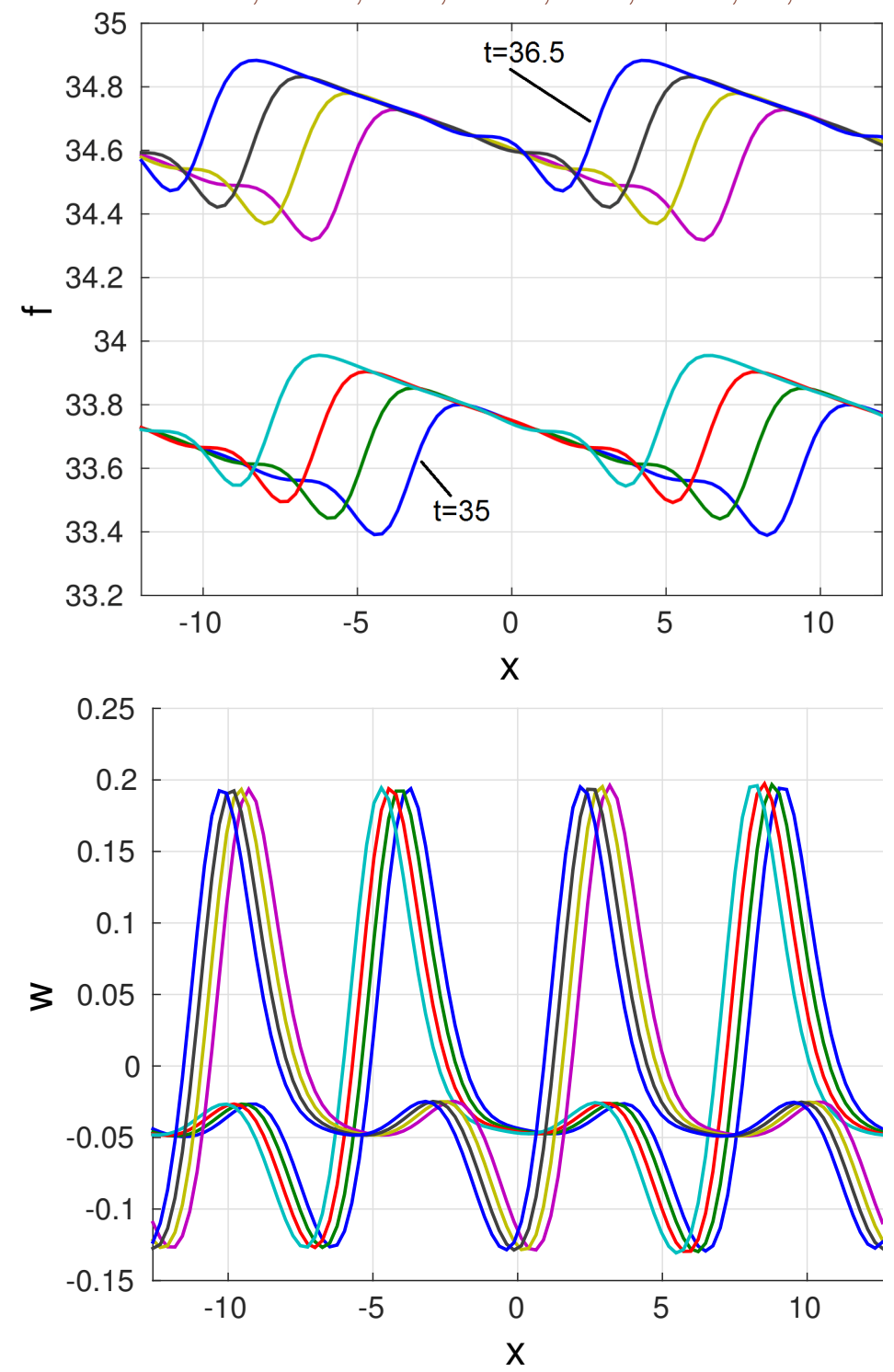


\section{References}

[1] S. J. Sherwin, L. Formaggia, J. Peiro and V. Franke. Computational modelling of 1D blood flow with variable mechanical properties and its application to the simulation of wave propagation in the human arterial system. Int. J. Numer. Meth. Fluids, 43:673-700, 2003. doi:10.1002/fld.543 C222

[2] C. Kleinstreuer. Biofluid Dynamics. Taylor and Francis, 2006. C222

[3] M. S. Olufsen, C. S. Peskin, W. Y. Kim, E. M. Pedersen, A. Nadim and J. Larsen. Numerical simulation and experimental validation of blood flow in arteries with structured-tree outflow conditions. Ann. Biomed. Eng., 28:1281-1299, 2000. doi:10.1114/1.1326031 C222

[4] K. S. Matthys, J. Alastruey, J. Peiro, A. W. Khir, P. Segers, P. R. Verdonck, K. H. Parker and S. J. Sherwin. Pulse wave propagation in a model human arterial network: Assessment of 1-D numerical simulations against in vitro measurements. J. Biomech., 40:3476-3486, 2007. doi:10.1016/j.jbiomech.2007.05.027 C222

[5] A. J. Roberts. A One-dimensional Introduction to Continuum Mechanics. World Scientific. Singapore, 1994. doi:10.1142/2496 C222

[6] D. V. Strunin, Fluid flow between active elastic plates. ANZIAM J., 50:C871-C883, 2009. doi:10.21914/anziamj.v50i0.1452 C222, C224

[7] D. V. Strunin, Autosoliton model of the spinning fronts of reaction. IMA J. Appl. Math., 63:163-177, 1999. doi:10.1093/imamat/63.2.163 $\mathrm{C} 223$

[8] R. Huang and Z. Suo. Wrinkling of a compressed elastic film on a viscous layer. J. Appl. Phys., 91:1135-1142, 2002. doi:10.1063/1.1427407 C224

[9] J. King. The isolation oxidation of silicon: The reaction-controlled case. SIAM J. Appl. Math. 49:1064-1080, 1989. doi:10.1137/0149064 C224 
[10] D. Ngo-Cong, N. Mai-Duy, W. Karunasena and T. Tran-Cong. Local moving least square-one-dimensional integrated radial basis function networks technique for incompressible viscous flows. Int. J. Numer. Meth. Fluids, 70:1443-1474, 2012. doi:10.1002/fld.3640 C227

[11] P. Le, N. Mai-Duy, T. Tran-Cong and G. Baker. A meshless modeling of dynamic strain localization in quasi-brittle materials using radial basis function networks, CMES-Comp. Model. Eng., 25:43-66, 2008. doi:10.3970/cmes.2008.025.043 C227

[12] N. Mai-Duy and T. Tran-Cong. Numerical solution of differential equations using multiquadric radial basis function networks, Neural Networks, 14:185-199, 2001. doi:10.1016/S0893-6080(00)00095-2 C227

\section{Author addresses}

1. F. Z. Ahmed, Faculty of Health, Engineering and Science, University of Southern Queensland, Queensland 4350, Australia. mailto:u1054915@umail.usq. edu.au

2. D. V. Strunin, Faculty of Health, Engineering and Science, University of Southern Queensland, Queensland 4350, Australia. http://www.sci.usq.edu.au/staff/strunin/ mailto:strunin@usq. edu.au

3. M. G. Mohammed, Department of Mathematics, University of Thi-Qar, Iraq.

mailto:w0102000@umail.usq. edu.au

4. R. P. Bhanot, Faculty of Health, Engineering and Science, University of Southern Queensland, Queensland 4350, Australia. mailto:w0077344@umail.usq. edu.au 